\title{
Total edge irregularity strength of quadruplet and quintuplet book graphs
}

\author{
Lucia Ratnasari $^{1 *}$, Sri Wahyuni $^{1}$, Yeni Susanti ${ }^{1}$, and Diah Junia Eksi Palupi ${ }^{1}$ \\ ${ }^{1}$ Department of Mathematics, Universitas Gadjah Mada, Yogyakarta, Indonesia
}

\begin{abstract}
Let $G=(V, E)$ be a finite, simple and undirected graph with a vertex set $V$ and an edge set $E$. An edge irregular total $k$-labelling is a function $f: V \cup E \rightarrow\{1,2, \ldots, k\}$ such that for any two different edges $x y$ and $x^{\prime} y^{\prime}$ in $E$, their weights are distinct. The weight of edge $x y$ is the sum of label of edge $x y$, labels of vertex $x$ and of vertex $y$. The minimum $k$ for which the graph $G$ admits an edge irregular total $k$-labelling is called the total edge irregularity strength of $G$, denoted by tes $(G)$. We have determined the total edge irregularity strength of book graphs, double book graphs and triple book graphs. In this paper, we show the exact value of the total edge irregularity strength of quadruplet book graphs and quintuplet book graphs.
\end{abstract}

\section{Introduction}

Graph labelling is a function of the set of integers to the set of elements on the graph (vertices, edges or both) with certain conditions [1]. Irregular edge $k$-labelling was introduced by Chartrand et al. [2] as a function of the set of edges to the set $\{1,2, \ldots, k\}$ such that any two different vertices in graph $G$ have different weights. Let $v$ be a vertex ; the weight of $v$ is the sum of labels of edges that are incident to vertex $v$. If graph $G$ can be labelled with an irregular edge $k$-labelling, then the minimum $k$ is called irregularity strength of $G$ (denoted by $s(G)$ ).

Bača et al. defined an edge irregular total $k$-labelling of graph $G$ as a function $f$ from the union of the set of vertices and the set of edges to the set $\{1,2, \ldots, k\}$ such that any two different edges of $G$ have different weights [3]. Let $x y$ be an edge. The weight of the edge $x y$ (denoted by $\omega_{f}(x y)$ ) is $\omega_{f}(x y)=f(x)+f(y)+f(x y)$. If the graph $G$ can be labelled with a total irregular $k$-labelling, then the minimum $k$ is called the total edge irregularity strength $G$ (denoted by tes $(G)$ ). In [3], Bača et al. have also given the lower bound of tes $(G)$.

Ivanco and Jendrol in [4] have determined tes of trees. Research on the tes of cyclic graphs for various graph classes is still being done. Several studies on the exact value of tes in some cyclic graphs, including some book graphs, have been conducted by [5-19].

In previous research [20], we have shown the tes of triple book graphs, and we have constructed the formula of an edge irregular total $k$-labelling of the first book, the second book and the third book. The results, the first book, the second book and the third book have

*Corresponding author: ratnasari.lucia@gmail.com 
different edge labelling formulas. In this research, we investigate the formula of an edge irregular total $k$-labelling and determine the tes of quadruplet and quintuplet book graphs.

\section{Main result}

We define the book graph, quadruplet book graph and quintuplet book graph as below:

Definition 2.1. Let $C_{m}^{i}, i=1,2, \ldots, n$ be cycle graphs and the vertices of $C_{m}^{i}$ be $V\left(C_{m}^{i}\right)=\{u, v\} \cup\left\{x_{i, j}: j=1, \ldots, m-2\right\}$ and the edges of $C_{m}^{i}$ be $E\left(C_{m}^{i}\right)=\left\{u v, u x_{i 1}, x_{i, j} x_{i, j+1}, x_{m-2} v: j=1, \ldots, m-3\right\}$.

A book graph $B_{n}\left(C_{m}\right)$ is a graph obtained from cycle graphs $C_{m}^{i}$ by merging edge uv from each cycle. The vertex set of $B_{n}\left(C_{m}\right)$ is $V\left(B_{n}\left(C_{m}\right)\right)=\{u, v\} \cup\left\{x_{i, j}: i=1, \ldots, n, j=1, \ldots, m-2\right\}$, and the edge set $i s$ $E\left(B_{n}\left(C_{m}\right)\right)=\{u v\} \cup\left\{u x_{i 1}, x_{i, j} x_{i, j+1}, x_{m-2} v: i=1, \ldots, n, j=1, \ldots, m-2\right\}$.

Furthermore, we define quadruplet book graphs.

Definition 2.2. Let $B_{n}^{q}\left(C_{m}\right), 1 \leq q \leq 4$ be the $q^{\text {th }}$ copy of book graph $B_{n}\left(C_{m}\right)$ as defined in Definition 2.1. Let the vertices of $B_{n}^{q}\left(C_{m}\right)$ be

$$
V\left(B_{n}^{q}\left(C_{m}\right)\right)=\left\{u^{q}, v^{q}\right\} \cup\left\{x_{i, j}^{q}: i=1, \ldots, n ; j=1, \ldots, m-2\right\} .
$$

A quadruplet book graph $4 B_{n}\left(C_{m}\right)$ is a graph obtained from four copies of book graph $B_{n}^{q}\left(C_{m}\right)$ by identifying vertex $v^{q}$ from book graph $B_{n}^{q}\left(C_{m}\right)$ as vertex $u^{q+1}$ from book graph $B_{n}^{q+1}\left(C_{m}\right)$ and renaming this vertex as $Z^{q}, 1 \leq q \leq 3$.

The vertex set of $4 B_{n}\left(C_{m}\right)$ is

$$
V\left(4 B_{n}\left(C_{m}\right)\right)=\left\{u^{1}, z^{1}, z^{2}, z^{3}, v^{4}\right\} \cup\left\{x_{i, j}^{1}, x_{i, j}^{2}, x_{i, j}^{3}, x_{i, j}^{4}: i=1, \ldots n ; j=1, \ldots, m-2\right\}
$$
and the edge set of $4 B_{n}\left(C_{m}\right)$ is

$$
\begin{aligned}
E\left(4 B_{n}\left(C_{m}\right)\right)= & \left\{u^{1} z^{1}, z^{1} z^{2}, z^{2} z^{3}, z^{3} v^{4}\right\} \cup \\
& \left\{\bigcup_{i=1}^{n} \bigcup_{j=1}^{m-2} u x_{i, 1}^{1}, x_{i, j}^{1} x_{i, j+1}^{1}, x_{i, m-2}^{1} z^{1}, \ldots, z^{3} x_{i, 1}^{4} x_{j, 1}^{4} x_{i, j+1}^{4}, x_{i, m-2}^{4} v^{4}\right\} .
\end{aligned}
$$

Similarly, we define quintuplet book graphs as follows:

Definition 2.3. Let $B_{n}^{q}\left(C_{m}\right), 1 \leq q \leq 5$ be the $q^{\text {th }}$ copy of book graph $B_{n}\left(C_{m}\right)$ as defined in Definition 2.1. Let the vertices of $B_{n}^{q}\left(C_{m}\right)$ be

$$
V\left(B_{n}^{q}\left(C_{m}\right)\right)=\left\{u^{q}, v^{q}\right\} \cup\left\{x_{i, j}^{q}: i=1, \ldots, n ; j=1, \ldots, m-2\right\} .
$$

A quintuplet book graph $5 B_{n}\left(C_{m}\right)$ is a graph obtained from five copies of book graph $B_{n}^{q}\left(C_{m}\right)$ by identifying vertex $v^{q}$ from book graph $B_{n}^{q}\left(C_{m}\right)$ as vertex $u^{q+1}$ from book graph $B_{n}^{q+1}\left(C_{m}\right)$ and renaming this vertex as $z^{q}, 1 \leq q \leq 4$.

The vertex set of $5 B_{n}\left(C_{m}\right)$ is

$$
\begin{aligned}
V\left(5 B_{n}\left(C_{m}\right)\right)= & \left\{u^{1}, z^{1}, z^{2}, z^{3}, z^{4}, v^{5}\right\} \\
& \cup\left\{x_{i, j}^{1}, x_{i, j}^{2}, x_{i, j}^{3}, x_{i, j}^{4}, x_{i, j}^{5}: i=1, \ldots, n ; j=1, \ldots, m-2\right\}
\end{aligned}
$$

and the edge set of $5 B_{n}\left(C_{m}\right)$ is

$$
\begin{aligned}
E\left(5 B_{n}\left(C_{m}\right)\right)= & \left\{u^{1} z^{1}, z^{1} z^{2}, z^{2} z^{3}, z^{3} z^{4}, z^{4} v^{5}\right\} \cup \\
& \left\{\bigcup_{i=1}^{n} \bigcup_{j=1}^{m-2} u x_{i, 1}^{1}, x_{i, j}^{1} x_{i, j+1}^{1}, x_{i, m-2}^{1} z^{1}, \ldots, z^{4} x_{i, 1}^{5} x_{j, 1}^{5} x_{i, j+1}^{5}, x_{i, m-2}^{5} v^{5}\right\} .
\end{aligned}
$$

We show the tes of quadruplet and of quintuplet book graphs in section 2.1. and section 2.2, respectively. 


\subsection{Total edge irregularity strength of quadruplet book graphs}

We determine the tes quadruplet book graphs as described in Theorem 2.4.

Theorem 2.4. Let $4 B_{n}\left(C_{m}\right)$ be a quadruplet book graph. Then the tes of $4 B_{n}\left(C_{m}\right)$ is $\left\lceil\frac{4(m-1) n+6}{3}\right\rceil$.

\section{Proof.}

A quadruplet book graph $4 B_{n}\left(C_{m}\right)$ has $m$ sides and $4 n$ sheets; therefore, from Definition 2.2. is obtained $\left|E\left(4 B_{n}\left(C_{m}\right)\right)\right|=4(m-1) n+4$. By lower bound in [2] (i.e., tes $(G) \geq$ $\left.\max \left\{\left\lceil\frac{|E|+2}{3}\right\rceil,\left\lceil\frac{\Delta(G)+1}{2}\right\rceil\right\}\right)$, we obtain that $\operatorname{tes}\left(4 B_{n}\left(C_{m}\right)\right) \geq\left\lceil\frac{4(m-1) n+6}{3}\right\rceil$ because any book graph $4\left(B_{n}\left(C_{m}\right)\right.$ has a maximum degree $\Delta\left(4\left(B_{n}\left(C_{m}\right)\right)=2(n+1)\right.$. By constructing an edge irregular total $k$-labelling for $4 B_{n}\left(C_{m}\right)$ with $k_{4}=\left\lceil\frac{4(m-1) n+6}{3}\right\rceil$ we can prove the upper bound.

We know from Definition 2.2. that $z^{1}=v^{1}=u^{2}, z^{2}=v^{2}=u^{3}, z^{3}=v^{3}=u^{4}$ and we define the vertex labelling $f$ in the following way:
$f\left(u^{1}\right)=k_{0}$,
$f\left(v^{q}\right)=k_{q}$,
$1 \leq q \leq 4$
$f\left(x_{i, j}^{q}\right)=k_{q-1}+i+j-2$,
$1 \leq i \leq n, 1 \leq j \leq y$
$f\left(x_{i, j}^{q}\right)=k_{q-1}+\frac{3 j-m+a}{6} n+\frac{m-j-b}{2}+i$
$1 \leq i \leq n, j=y+p, p=1,3, \ldots, 2 y-5$
$f\left(x_{i, j}^{q}\right)=k_{q-1}+\frac{3 j-m-c}{6} n+\frac{m-j-d}{2}+i, 1 \leq i \leq n, j=y+p, p=2,4, \ldots, 2 y-e$
$f\left(x_{i, j}^{q}\right)=k_{q-1}+\frac{3 j-m+3}{6} n+i$,
if $m=0(\bmod 3), 1 \leq i \leq r_{q}, j=m-3$
$f\left(x_{i, j}^{q}\right)=k_{q}$,
$f\left(x_{i, m-2}^{q}\right)=k_{q}$,
$1 \leq i \leq n$,
with $k_{0}=1, k_{q}=\left\lceil\frac{q((m-1) n+1)+2}{3}\right\rceil, r_{q}=k_{q}-\frac{m-3}{3} n+k_{q-1}$ and $q=1$ for the first book, $q=2$ for the second book, $q=3$ for the third book, $q=4$ for the fourth book and
i. $\quad a=3, b=5, c=0, d=2, e=4, y=\frac{m}{3} \quad$ for $m=0(\bmod 3)$,
ii. $\quad a=1, b=5, c=2, d=2, e=6, y=\frac{m+2}{3} \quad$ for $m=1(\bmod 3)$,
iii. $\quad a=2, b=6, c=1, d=3, e=6, y=\frac{m+1}{3} \quad$ for $m=2(\bmod 3)$.

if $m=0(\bmod 3), r_{q}+1 \leq i \leq n, j=m-3$

We have defined the edge labelling $f$ for the first book, the second book and the third book in [20]. We prove the edge labelling $f$ of the fourth book for three different cases.

\section{For $m \equiv 0 \bmod 3$.}

The edge labelling $f$ for the fourth book is defined as follows:

$$
\begin{aligned}
& f\left(u^{4}, v^{4}\right)= \begin{cases}k_{3}+n-\left(\frac{2 n+2}{3}\right), & n=2(\bmod 3) \\
k_{3}+n-\left(\frac{2 n}{3}\right), & n=0(\bmod 3) \\
k_{3}+n-\left(\frac{2 n+1}{3}\right), & n=1(\bmod 3)\end{cases} \\
& f\left(u^{4} x_{i, 1}^{4}\right)=k_{3} \text {, } \\
& 1 \leq i \leq n \\
& f\left(x_{i, j}^{4} x_{i, j+1}^{4}\right)=k_{3}+j n-2 j+2-i \text {, } \\
& 1 \leq i \leq n, 1 \leq j \leq \frac{m-3}{3} \\
& f\left(x_{i, j}^{4} x_{i, j+1}^{4}\right)=k_{3}+\frac{m-3}{3} n-m+j+5-i, \quad 1 \leq i \leq n, j=\frac{m}{3} \\
& f\left(x_{i, j}^{4} x_{i, j+1}^{4}\right)=k_{3}+\frac{m-3}{3} n-m+j+4-i, \quad 1 \leq i \leq n, \quad \frac{m+3}{3} \leq j \leq m-5
\end{aligned}
$$




$$
\begin{aligned}
& f\left(x_{i, j}^{4} x_{i, j+1}^{4}\right)=k_{3}+\frac{m-3}{3} n-i+1, \quad 1 \leq i \leq r_{4}, j=m-4 \\
& f\left(x_{i, j}^{4} x_{i, j+1}^{4}\right)=2 k_{3}-k_{4}+2 \frac{m-3}{3} n, \quad r_{4}+1 \leq i \leq n, j=m-4 \\
& f\left(x_{i, j}^{4} x_{i, j+1}^{4}\right)= \begin{cases}k_{3}+\frac{m-3}{3} n-\frac{(2 n+2)}{3}, & 1 \leq i \leq r_{4}, j=m-3, n=2(\bmod 3) \\
k_{3}+\frac{m-3}{3} n-\frac{(2 n)}{3}, & 1 \leq i \leq r_{4}, j=m-3, n=0(\bmod 3) \\
k_{3}+\frac{m-3}{3} n-\frac{(2 n+1)}{3}, & 1 \leq i \leq r_{4}, j=m-3, n=1(\bmod 3)\end{cases} \\
& f\left(x_{i, j}^{4} x_{i, j+1}^{4}\right)= \begin{cases}k_{3}+\frac{m-3}{3} n-\frac{(2 n+2)}{3}+p, & r_{4} \leq i \leq n, j=m-3, n=2(\bmod 3) \\
k_{3}+\frac{m-3}{3} n-\frac{(2 n)}{3}+p, & r_{4} \leq i \leq n, j=m-3, n=0(\bmod 3) \\
k_{3}+\frac{m-3}{3} n-\frac{(2 n+1)}{3}+p, & r_{4} \leq i \leq n, j=m-3, n=1(\bmod 3)\end{cases} \\
& \text { with } 1 \leq p \leq n-r_{4} \text { and } r_{4}=k_{4}-\frac{(m-3)}{3} n+k_{3} \\
& f\left(x_{i, j}^{4} v^{4}\right)= \begin{cases}k_{3}+\frac{m-6}{3} n-2\left(\frac{n+1}{3}\right)+i-2, & 1 \leq i \leq n, j=m-2, n=2(\bmod 3) \\
k_{3}+\frac{m-6}{3} n-2 \frac{(n+3)}{3}+i-2, & 1 \leq i \leq n, j=m-2, n=0(\bmod 3) \\
k_{3}+\frac{m-6}{3} n-2 \frac{(n+2)}{3}+i-2, & 1 \leq i \leq n, j=m-2, n=1(\bmod 3)\end{cases}
\end{aligned}
$$

Under the labelling $f$, we have the weight of the edges of the fourth book graph as below:

$\omega_{f}\left(u^{4}, v^{4}\right)= \begin{cases}2 k_{3}+k_{4}+\left(\frac{n-2}{3}\right), & n=2(\bmod 3) \\ 2 k_{3}+k_{4}+\left(\frac{n}{3}\right), & n=0(\bmod 3) \\ 2 k_{3}+k_{4}+\left(\frac{n-1}{3}\right), & n=1(\bmod 3)\end{cases}$

$\omega_{f}\left(u^{4} x_{i, 1}^{4}\right)=3 k_{3}+i-1$,

$\begin{array}{ll}\omega_{f}\left(x_{i, j}^{4} x_{i, j+1}^{4}\right)=3 k_{3}+j n+i-1, & 1 \leq j \leq \frac{m-3}{3} \\ \omega_{f}\left(x_{i, j}^{4} x_{i, j+1}^{4}\right)=3 k_{3}+j n+i, & \frac{m}{3} \leq j \leq m-4\end{array}$

$\omega_{f}\left(x_{i, j}^{4} x_{i, j+1}^{4}\right)= \begin{cases}2 k_{3}+k_{4}+i+\frac{(2 m-8) n-1}{3}, & j=m-3, n=2(\bmod 3) \\ 2 k_{3}+k_{4}+i+\frac{(2 m-8) n}{3}, & j=m-3, n=0(\bmod 3) \\ 2 k_{3}+k_{4}+i+\frac{(2 m-8) n-2}{3}, & j=m-3, n=1(\bmod 3)\end{cases}$

$\omega_{f}\left(x_{i, j}^{4} v^{4}\right)= \begin{cases}k_{3}+2 k_{4}+i+\frac{(2 m-4) n-2}{3}, & j=m-3, n=2(\bmod 3) \\ k_{3}+2 k_{4}+i+\frac{(2 m-4) n}{3}, & j=m-3, n=0(\bmod 3) \\ k_{3}+2 k_{4}+i+\frac{(2 m-4) n-1}{3}, & j=m-3, n=1(\bmod 3)\end{cases}$

with $1 \leq i \leq n$.

\section{For $m \equiv 1 \bmod 3$.}

The edge labelling $f$ for the fourth book is defined as follows:

$f\left(u^{4}, v^{4}\right)=k_{3}+n$,

$f\left(u^{4} x_{i, 1}^{4}\right)=k_{3}$,

$1 \leq i \leq n$

$f\left(x_{i, j}^{4} x_{i, j+1}^{4}\right)=k_{3}+j n-2 j-i+2$,

$1 \leq i \leq n, 1 \leq j \leq \frac{m-1}{3}$

$f\left(x_{i, j}^{4} x_{i, j+1}^{4}\right)=k_{3}+\frac{m-1}{3} n+j-m-i+4$,

$1 \leq i \leq n, \quad \frac{m+2}{3} \leq j \leq m-4$

$f\left(x_{i, j}^{4} x_{i, j+1}^{4}\right)=k_{3}+\frac{m-4}{3} n+1$,

$1 \leq i \leq n, j=m-3$ 


$$
f\left(x_{i, j}^{4} v^{4}\right)=k_{3}+\frac{m-4}{3} n+i, \quad 1 \leq i \leq n, j=m-2 .
$$

Under the labelling $f$, we have the weight of the edges of the fourth book graph as below:

$\omega_{f}\left(u^{4} x_{i, 1}^{4}\right)=3 k_{3}+i-1$,

$\omega_{f}\left(x_{i, j}^{4} x_{i, j+1}^{4}\right)=3 k_{3}+j n+i-1$,

$1 \leq j \leq \frac{m-1}{3}$

$\omega_{f}\left(u^{4}, v^{4}\right)=2 k_{3}+k_{4}+n$,

$\omega_{f}\left(x_{i, j}^{4} x_{i, j+1}^{4}\right)=3 k_{3}+j n+i$,

$\frac{m+2}{3} \leq j \leq m-4$

$\omega_{f}\left(x_{i, j}^{4} x_{i, j+1}^{4}\right)=2 k_{3}+k_{4}+2\left(\frac{m-4}{3}\right) n+i$

$j=m-3$

$\omega_{f}\left(x_{i, j}^{4} v^{4}\right)=k_{3}+2 k_{4}+\frac{m-4}{3} n+i$,

$j=m-2$,

with $1 \leq i \leq n$.

\section{For $m \equiv 2 \bmod 3$.}

The edge labelling $f$ for the fourth book is defined as below:

$$
\begin{aligned}
& \left(k_{3}+n-\left(\frac{n+1}{3}\right), \quad n=2(\bmod 3)\right. \\
& f\left(u^{4}, v^{4}\right)= \begin{cases}k_{3}+n-\left(\frac{n}{3}\right), & n=0(\bmod 3)\end{cases} \\
& k_{3}+n-\left(\frac{n+2}{3}\right), \quad n=1(\bmod 3) \\
& f\left(x_{i, j}^{4} x_{i, j+1}^{4}\right)=k_{3}+j n-2 j-i+2, \quad 1 \leq i \leq n, 1 \leq j \leq \frac{m-2}{3} \\
& f\left(x_{i, j}^{4} x_{i, j+1}^{4}\right)=k_{3}+\frac{m-2}{3} n+j-m-i+5, \quad 1 \leq i \leq n, \frac{m+1}{3} \leq j \leq m-4 \\
& f\left(x_{i, j}^{4} x_{i, j+1}^{4}\right)= \begin{cases}k_{3}+\frac{m-2}{3} n-\left(\frac{n+1}{3}\right), & 1 \leq i \leq n, j=m-3, n=2(\bmod 3) \\
k_{3}+\frac{m-2}{3} n-\left(\frac{n}{3}\right), & 1 \leq i \leq n, j=m-3, n=0(\bmod 3) \\
k_{3}+\frac{m-2}{3} n-\left(\frac{n+2}{3}\right), & 1 \leq i \leq n, j=m-3, n=1(\bmod 3)\end{cases} \\
& f\left(x_{i, j}^{4} v^{4}\right)= \begin{cases}k_{3}+\frac{m-2}{3} n-\left(\frac{n+1}{3}\right)+i, & j=m-2, n=2(\bmod 3) \\
k_{3}+\frac{m-2}{3} n-\left(\frac{n}{3}\right)+i, & j=m-2, n=0(\bmod 3) \\
k_{3}+\frac{m-2}{3} n-\left(\frac{n+2}{3}\right)+i, & j=m-2, n=1(\bmod 3) .\end{cases}
\end{aligned}
$$

Under the labelling $f$, we have the weight of the edges of the fourth book graph as below:

$\omega_{f}\left(u^{4} x_{i, 1}^{4}\right)=3 k_{3}+i-1$,

$$
\begin{aligned}
& \omega_{f}\left(x_{i, j}^{4} x_{i, j+1}^{4}\right)=3 k_{3}+j n+i-1 \text {, } \\
& \omega_{f}\left(u^{4}, v^{4}\right)=\left\{\begin{array}{l}
2 k_{3}+k_{4}+n-\left(\frac{n+1}{3}\right), \\
2 k_{3}+k_{4}+n-\left(\frac{n}{3}\right), \\
2 k_{3}+k_{4}+n-\left(\frac{n+2}{3}\right),
\end{array}\right. \\
& 1 \leq j \leq \frac{m-1}{3} \\
& n=2(\bmod 3) \\
& n=0(\bmod 3) \\
& n=1(\bmod 3) \\
& \omega_{f}\left(x_{i, j}^{4} x_{i, j+1}^{4}\right)=3 k_{3}+j n+i \text {, } \\
& \frac{m+2}{3} \leq j \leq m-4 \\
& \omega_{f}\left(x_{i, j}^{4} x_{i, j+1}^{4}\right)= \begin{cases}2 k_{3}+k_{4}+i+\frac{(2 m-8) n-1}{3}, & j=m-3, n=2(\bmod 3) \\
2 k_{3}+k_{4}+i+\frac{(2 m-8) n}{3}, & j=m-3, n=0(\bmod 3) \\
2 k_{3}+k_{4}+i+\frac{(2 m-8) n-2}{3}, & j=m-3, n=1(\bmod 3)\end{cases}
\end{aligned}
$$


$\omega_{f}\left(x_{i, j}^{4} v^{4}\right)= \begin{cases}k_{3}+2 k_{4}+i+\frac{(2 m-4) n-2}{3}, & j=m-3, n=2(\bmod 3) \\ k_{3}+2 k_{4}+i+\frac{(2 m-4) n}{3}, & j=m-3, n=0(\bmod 3) \\ k_{3}+2 k_{4}+i+\frac{(2 m-4) n-1}{3}, & j=m-3, n=1(\bmod 3),\end{cases}$

with $1 \leq i \leq n$.

For Cases 1, 2 and 3, under the labelling $f$, the weights of edges of $4 B_{n}\left(C_{m}\right)$ constitute the set $\{3, \ldots, 4(m-1) n+6\}$. This shows that the weights of all the edges of $4 B_{n}\left(C_{m}\right)$ are different and thus tes $\left(4 B_{n}\left(C_{m}\right)\right)=\left\lceil\frac{4(m-1) n+6}{3}\right\rceil$.

\subsection{Total edge irregularity strength of quintuplet book graphs}

In this section we discuss the tes of quintuplet book graphs.

Theorem 2.5. Let $5 B_{n}\left(C_{m}\right)$ be a quintuplet book graph. Then the tes of $\left(5 B_{n}\left(C_{m}\right)\right)$ is $\left\lceil\frac{5(m-1) n+7}{3}\right\rceil$.

\section{Proof.}

A quintuplet book graph $5 B_{n}\left(C_{m}\right)$ has $m$ sides and $5 n$ sheets; therefore from Definition 2.3. is obtained $\left|E\left(5 B_{n}\left(C_{m}\right)\right)\right|=5(m-1) n+7$. Bača et al. in [2] give the lower bound for $\operatorname{tes}(\mathrm{G})$; that is, tes $(\mathrm{G}) \geq \max \left\{\left\lceil\frac{|E|+2}{3}\right\rceil,\left\lceil\frac{\Delta(G)+1}{2}\right]\right\}$. Any book graph $5\left(B_{n}\left(C_{m}\right)\right.$ has maximum degree $\Delta\left(5\left(B_{n}\left(C_{m}\right)\right)=2(n+1)\right.$, so we have tes $\left(5 B_{n}\left(C_{m}\right)\right) \geq\left\lceil\frac{5(m-1) n+7}{3}\right\rceil$. To prove the upper bound, we construct an edge irregular total $k$-labelling for $5 B_{n}\left(C_{m}\right)$ with $k_{5}=$ $\left\lceil\frac{5(m-1) n+7}{3}\right\rceil$ for three different cases.

We know from Definition 2.3. that $z^{1}=v^{1}=u^{2}, z^{2}=v^{2}=u^{3}, z^{3}=v^{3}=u^{4}, z^{4}=v^{4}$ $=u^{5}$, and we define the vertex labelling $f$ similarly as in Theorem 2.4.

with $q=1$ for the first book, $q=2$ for the second book, $q=3$ for the third book, $q=4$ for the fourth book, $q=5$ for the fifth book; we define $k_{0}=1, k_{q}=\left\lceil\frac{q((m-1) n+1)+2}{3}\right\rceil$.

For the three cases, we define the edge labelling $f$ for the fifth book as follows:

\section{For $m \equiv 0 \bmod 3$.}

The edge labelling $f$ for the fifth book is defined in the following way:

$$
\begin{aligned}
& f\left(u^{5}, v^{5}\right)= \begin{cases}k_{4}+n-\left(\frac{2 n+2}{3}\right), & n=2(\bmod 3) \\
k_{4}+n-\left(\frac{2 n}{3}\right), & n=0(\bmod 3) \\
k_{4}+n-\left(\frac{2 n+1}{3}\right), & n=1(\bmod 3)\end{cases} \\
& f\left(w^{5} x_{i, 1}^{5}\right)= \begin{cases}k_{4}-1 & 1 \leq i \leq n, n=2(\bmod 3) \\
k_{4}+1, & 1 \leq i \leq n, n=0(\bmod 3) \\
k_{4}, & 1 \leq i \leq n, n=1(\bmod 3)\end{cases} \\
& f\left(x_{i, j}^{5} x_{i, j+1}^{5}\right)= \begin{cases}k_{4}+j n-2 j-i+1, & 1 \leq i \leq n, \frac{m+3}{3} \leq j \leq m-5, n=2(\bmod 3) \\
k_{4}+j n-2 j-i+3, & 1 \leq i \leq n, \frac{m+3}{3} \leq j \leq m-5, n=0(\bmod 3) \\
k_{4}+j n-2 j-i+2, & 1 \leq i \leq n, \frac{m+3}{3} \leq j \leq m-5, n=1(\bmod 3)\end{cases}
\end{aligned}
$$




$$
\begin{aligned}
& f\left(x_{i, j}^{5} x_{i, j+1}^{5}\right)= \begin{cases}k_{4}+\frac{m-3}{3} n-m+j-i+2, & 1 \leq i \leq r_{2}, j=m-4, n=2(\bmod 3) \\
k_{4}+\frac{m-3}{3} n-m+j-i+4, & 1 \leq i \leq r_{2}, j=m-4, n=0(\bmod 3) \\
k_{4}+\frac{m-3}{3} n-m+j-i+3, & 1 \leq i \leq r_{2}, j=m-4, n=1(\bmod 3)\end{cases} \\
& f\left(x_{i, j}^{5} x_{i, j+1}^{5}\right)\left\{\begin{array}{l}
2 k_{4}-k_{5}+2 \frac{m-3}{3} n-m+j+2, \quad r_{5}+1 \leq i \leq n, j=m-4, n=2(\bmod 3) \\
2 k_{4}-k_{5}+2 \frac{m-3}{3} n-m+j+4, r_{5}+1 \leq i \leq n, j=m-4, n=0(\bmod 3) \\
2 k_{4}-k_{5}+2 \frac{m-3}{3} n-m+j+3, r_{5}+1 \leq i \leq n, j=m-4, n=1(\bmod 3)
\end{array}\right. \\
& f\left(x_{i, j}^{5} x_{i, j+1}^{5}\right)= \begin{cases}k_{4}+\frac{m-3}{3} n-\frac{2 n+2}{3}, & 1 \leq i \leq r_{5}, j=m-3, n=2(\bmod 3) \\
k_{4}+\frac{m-3}{3} n-\frac{2 n}{3}, & 1 \leq i \leq r_{5}, j=m-3, n=0(\bmod 3) \\
k_{4}+\frac{m-3}{3} n-\frac{2 n+1}{3}, & 1 \leq i \leq r_{5}, j=m-3, n=1(\bmod 3)\end{cases} \\
& f\left(x_{i, j}^{5} x_{i, j+1}^{5}\right)= \begin{cases}k_{4}+\frac{m-3}{3} n-\frac{(2 n+2)}{3}+p, & r_{5}+1 \leq i \leq n, j=m-3, n=2(\bmod 3) \\
k_{4}+\frac{m-3}{3} n-\frac{(2 n)}{3}+p, & r_{5}+1 \leq i \leq n, j=m-3, n=0(\bmod 3) \\
k_{4}+\frac{m-3}{3} n-\frac{(2 n+1)}{3}+p, & r_{5}+1 \leq i \leq n, j=m-3, n=1(\bmod 3)\end{cases} \\
& \text { with } 1 \leq p \leq n-r_{5} \text { and } r_{5}=k_{5}-\frac{(m-3)}{3} n+k_{4} \\
& f\left(x_{i, j}^{5} v^{5}\right)= \begin{cases}k_{4}+\frac{m-6}{3} n+2 \frac{n+1}{3}+i, & 1 \leq i \leq n, j=m-2, n=2(\bmod 3) \\
k_{4}+\frac{m-6}{3} n+2 \frac{n+3}{3}+i, & 1 \leq i \leq n, j=m-2, n=0(\bmod 3) \\
k_{4}+\frac{m-6}{3} n+2 \frac{n+2}{3}+i, & 1 \leq i \leq n, \quad j=m-2, n=1(\bmod 3)\end{cases}
\end{aligned}
$$

Under the labelling $f$, we have the weights of the edges of the fifth book graph as follows:

$$
\begin{aligned}
& \omega_{f}\left(u^{5} x_{i, 1}^{5}\right)= \begin{cases}3 k_{4}+i-2, & n=2(\bmod 3) \\
3 k_{4}+i, & n=0(\bmod 3) \\
3 k_{4}+i-1, & n=1(\bmod 3)\end{cases} \\
& \omega_{f}\left(x_{i, j}^{5} x_{i, j+1}^{5}\right)= \begin{cases}3 k_{4}+j n+i-2, & 1 \leq j \leq \frac{m-2}{3}, n=2(\bmod 3) \\
3 k_{4}+j n+i, & 1 \leq j \leq \frac{m-2}{3}, n=0(\bmod 3) \\
3 k_{4}+j n+i-1, & 1 \leq j \leq \frac{m-2}{3}, n=1(\bmod 3)\end{cases} \\
& \omega_{f}\left(u^{5}, v^{5}\right)= \begin{cases}2 k_{4}+k_{5}-\left(\frac{n-2}{3}\right), & n=2(\bmod 3) \\
2 k_{4}+k_{5}-\left(\frac{n}{3}\right), & n=0(\bmod 3) \\
2 k_{4}+k_{5}-\left(\frac{n-1}{3}\right), & n=1(\bmod 3)\end{cases} \\
& \omega_{f}\left(x_{i, j}^{5} x_{i, j+1}^{5}\right)= \begin{cases}3 k_{4}+j n+i-1, & \frac{m}{3} \leq j \leq m-4, n=2(\bmod 3) \\
3 k_{4}+j n+i+1, & \frac{m}{3} \leq j \leq m-4, n=0(\bmod 3) \\
3 k_{4}+j n+i, & \frac{m}{3} \leq j \leq m-4, n=1(\bmod 3)\end{cases} \\
& \omega_{f}\left(x_{i, j}^{5} x_{i, j+1}^{5}\right)= \begin{cases}2 k_{4}+k_{5}+i+\frac{(2 m-8) n-2}{3}, & j=m-3, n=2(\bmod 3) \\
2 k_{4}+k_{5}+i+\frac{(2 m-8) n}{3}, & j=m-3, n=0(\bmod 3) \\
2 k_{4}+k_{5}+i+\frac{(2 m-8) n-1}{3}, & j=m-3, n=1(\bmod 3)\end{cases}
\end{aligned}
$$


$\omega_{f}\left(x_{i, j}^{5} v^{5}\right)= \begin{cases}k_{4}+2 k_{5}+i+\frac{(m-4) n-2}{3}, & j=m-3, n=2(\bmod 3) \\ k_{4}+2 k_{5}+i+\frac{(m-4) n}{3}, & j=m-3, n=0(\bmod 3) \\ k_{4}+2 k_{5}+i+\frac{(m-4) n-4}{3}, & j=m-3, n=1(\bmod 3),\end{cases}$

with $1 \leq i \leq n$.

\section{For $m \equiv 1 \bmod 3$.}

We construct the edge labelling $f$ for the fifth book as follows:

$f\left(u^{5}, v^{5}\right)=k_{4}+n$,

$f\left(u^{5} x_{i, 1}^{5}\right)=k_{4}+1$,

$1 \leq i \leq n$

$f\left(x_{i, j}^{5} x_{i, j+1}^{5}\right)=k_{4}+j n-2 j+3-i$,

$1 \leq i \leq n, 1 \leq j \leq \frac{m-1}{3}$

$f\left(x_{i, j}^{5} x_{i, j+1}^{5}\right)=k_{4}+\frac{m-1}{3} n+j+5-m-i$,

$1 \leq i \leq n, \quad \frac{m+2}{3} \leq j \leq m-4$

$f\left(x_{i, j}^{5} x_{i, j+1}^{5}\right)=k_{3}+\frac{m-4}{3} n+1$,

$1 \leq i \leq n, j=m-3$

$f\left(x_{i, j}^{5} v^{5}\right)=k_{3}+\frac{m-4}{3} n+i-1$,

$1 \leq i \leq n, j=m-2$.

Under the labelling $f$, we have the weights of the edges of the fifth book graph as follows:

$\omega_{f}\left(u^{5} x_{i, 1}^{5}\right)=3 k_{4}+i$,

$\omega_{f}\left(x_{i, j}^{5} x_{i, j+1}^{5}\right)=3 k_{4}+j n+i$,

$1 \leq j \leq \frac{m-1}{3}$

$\omega_{f}\left(u^{5}, v^{5}\right)=2 k_{4}+k_{5}+n$,

$\omega_{f}\left(x_{i, j}^{5} x_{i, j+1}^{5}\right)=3 k_{4}+j n+i+1$,

$\frac{m+2}{3} \leq j \leq m-4$

$\omega_{f}\left(x_{i, j}^{5} x_{i, j+1}^{5}\right)=2 k_{4}+k_{5}+2\left(\frac{m-4}{3}\right) n+i$,

$j=m-3$

$\omega_{f}\left(x_{i, j}^{5} v^{5}\right)=k_{4}+2 k_{5}+\frac{m-4}{3} n+i-1$,

$j=m-2$,

with $1 \leq i \leq n$.

\section{For $m \equiv 2 \bmod 3$.}

We define the edge labelling $f$ for the fifth book as below:

$$
\begin{aligned}
& f\left(u^{5}, v^{5}\right)= \begin{cases}k_{4}+n-\left(\frac{n+1}{3}\right), & n=2(\bmod 3) \\
k_{4}+n-\left(\frac{n}{3}\right), & n=0(\bmod 3) \\
k_{4}+n-\left(\frac{n+2}{3}\right), & n=1(\bmod 3)\end{cases} \\
& f\left(u^{5} x_{i, 1}^{5}\right)= \begin{cases}k_{4}, & 1 \leq i \leq n, n=2(\bmod 3) \\
k_{4}+1, & 1 \leq i \leq n, n=0(\bmod 3) \\
k_{4}-1, & 1 \leq i \leq n, n=1(\bmod 3)\end{cases} \\
& f\left(x_{i, j}^{5} x_{i, j+1}^{5}\right)=\left\{\begin{array}{ll}
k_{4}+j n-2 j-i+2, & 1 \leq i \leq n, 1 \leq j \leq \frac{m-2}{3}, n=2(\bmod 3) \\
k_{4}+j n-2 j-i+3, & 1 \leq i \leq n, 1 \leq j \leq \frac{m-2}{3}, n=0(\bmod 3) \\
k_{4}+j n-2 j-i+1, & 1 \leq i \leq n, 1 \leq j \leq \frac{m-2}{3}, n=1(\bmod 3)
\end{array}\right) \\
& f\left(x_{i, j}^{5} x_{i, j+1}^{5}\right)= \begin{cases}k_{4}+\frac{m-2}{3} n+j+5-m-i, & \frac{m+1}{3} \leq j \leq m-4, n=2(\bmod 3) \\
k_{4}+\frac{m-2}{3} n+j+6-m-i, & \frac{m+1}{3} \leq j \leq m-4, n=0(\bmod 3) \\
k_{4}+\frac{m-2}{3} n+j+4-m-i, & \frac{m+1}{3} \leq j \leq m-4, n=1(\bmod 3)\end{cases}
\end{aligned}
$$




$$
\begin{gathered}
f\left(x_{i, j}^{5} x_{i, j+1}^{5}\right)= \begin{cases}k_{4}+\frac{m-2}{3} n-\left(\frac{n+1}{3}\right), & 1 \leq i \leq n, j=m-3, n=2(\bmod 3) \\
k_{4}+\frac{m-2}{3} n-\left(\frac{n}{3}\right), & 1 \leq i \leq n, j=m-3, n=0(\bmod 3) \\
k_{4}+\frac{m-2}{3} n-\left(\frac{n+2}{3}\right), & 1 \leq i \leq n, j=m-3, n=1(\bmod 3)\end{cases} \\
f\left(x_{i, j}^{5} v^{5}\right)= \begin{cases}k_{4}+\frac{m-2}{3} n-2\left(\frac{n+1}{3}\right)+i, & 1 \leq i \leq n, j=m-2, n=2(\bmod 3) \\
k_{4}+\frac{m-2}{3} n-2\left(\frac{n}{3}\right)+i, & 1 \leq i \leq n, j=m-2, n=0(\bmod 3) \\
k_{4}+\frac{m-2}{3} n-2\left(\frac{n+2}{3}\right)+i, & 1 \leq i \leq n \quad j=m-2, n=1(\bmod 3) .\end{cases}
\end{gathered}
$$

Under the labelling $f$, we have the weights of the edges of the fifth book graph as below:

$$
\begin{aligned}
& \omega_{f}\left(u^{5} x_{i, 1}^{5}\right)= \begin{cases}3 k_{4}+i-1, & n=2(\bmod 3) \\
3 k_{4}+i, & n=0(\bmod 3) \\
3 k_{4}+i-2, & n=1(\bmod 3)\end{cases} \\
& \omega_{f}\left(x_{i, j}^{5} x_{i, j+1}^{5}\right)= \begin{cases}3 k_{4}+j n+i-1, & 1 \leq j \leq \frac{m-2}{3}, n=2(\bmod 3) \\
3 k_{4}+j n+i, & 1 \leq j \leq \frac{m-2}{3}, n=0(\bmod 3) \\
3 k_{4}+j n+i-2, & 1 \leq j \leq \frac{m-2}{3}, n=1(\bmod 3)\end{cases} \\
& \omega_{f}\left(u^{5}, v^{5}\right)= \begin{cases}2 k_{4}+k_{5}+n-\left(\frac{n+1}{3}\right), & n=2(\bmod 3) \\
2 k_{4}+k_{5}+n-\left(\frac{n}{3}\right), & n=0(\bmod 3) \\
2 k_{4}+k_{5}+n-\left(\frac{n+2}{3}\right), & n=1(\bmod 3)\end{cases} \\
& \omega_{f}\left(x_{i, j}^{5} x_{i, j+1}^{5}\right)= \begin{cases}3 k_{4}+j n+i, & \frac{m+1}{3} \leq j \leq m-4, n=2(\bmod 3) \\
3 k_{4}+j n+i+1, & \frac{m+1}{3} \leq j \leq m-4, n=0(\bmod 3) \\
3 k_{4}+j n+i-1, & \frac{m+1}{3} \leq j \leq m-4, n=1(\bmod 3)\end{cases} \\
& \omega_{f}\left(x_{i, j}^{5} x_{i, j+1}^{5}\right)=\left\{\begin{array}{ll}
2 k_{4}+k_{5}+i+\frac{(2 m-8) n-1}{3}, & j=m-3, n=2(\bmod 3) \\
2 k_{4}+k_{5}+i+\frac{(2 m-8) n}{3}, & j=m-3, n=0(\bmod 3) \\
2 k_{4}+k_{5}+i+\frac{(2 m-8) n-2}{3}, & j=m-3, n=1(\bmod 3)
\end{array}\right) \\
& \omega_{f}\left(x_{i, j}^{5} v^{5}\right)= \begin{cases}k_{4}+2 k_{5}+i+\frac{(m-4) n-2}{3}, & j=m-3, n=2(\bmod 3) \\
k_{4}+2 k_{5}+i+\frac{(m-4) n}{3}, & j=m-3, n=0(\bmod 3)\end{cases}
\end{aligned}
$$

with $1 \leq i \leq n$.

Under the labelling $f$, the weights of edges of $5 B_{n}\left(C_{m}\right)$ for cases 1,2 and 3 constitute the set $\{3, \ldots, 5(m-1) n+7\}$. This shows that the weights of all the edges of $\left(5 B_{n}\left(C_{m}\right)\right.$ are different and tes $\left(5 B_{n}\left(C_{m}\right)\right)=\left\lceil\frac{5(m-1) n+7}{3}\right\rceil$. 


\section{Conclusion}

We have constructed an edge irregular total $k$-labelling for the fourth book and the fifth book. We found that the total edge irregularity strength of quadruplet book graphs is tes $4 B_{n}\left(C_{m}\right)=$ $\left\lceil\frac{4(m-1) n+6}{3}\right\rceil$ and of quintuplet book graphs is tes $5 B_{n}\left(C_{m}\right)=\left\lceil\frac{5(m-1) n+7}{3}\right\rceil$.

Based on the labelling, we see that there is a similarity labelling for the first book with the fourth book and for the second book with the fifth book as well. By the results, it is reasonable to find further formulations of the labelling for general cases.

This work was supported by Doctoral Research Grant, Kementerian Riset dan Teknology, Indonesian Government, No.3117/UN1.DITLIT/DIT-LIT/PT/2020.

\section{References}

1. J.A. Gallian, Electron. J. Comb. (2019)

2. G. Chartrand, M. Jacobson, J. Lehel, O. Oellermann, S. Ruiz, F.Saba, Congr. Numer. 64, $197-210$ (1988)

3. M. Bača, S. Jendrol, M. Miller, J. Ryan, Discrete Math. 307, 1378-1388 (2007)

4. J. Ivanco, S. Jendrol, Discuss. Math. Graph Theory 26, 449 - 456 (2006)

5. S. Jendrol, J. Miškuf, R. Sotak, Electron. Notes Discret. Math. 28, 281 - 285 (2007)

6. Nurdin, A.N.M. Salman, E.T. Baskoro, J. Comb. Math. Comb. Comput. 65, 163 - 175 (2008)

7. T. Chunling, L. Xiaohui, Y. Yuansheng, W. Liping, Indian J. Pure Appl, Math. 40(3), 155 - 181 (2009)

8. M. Bača, M.K. Siddiqui, Appl Math. Comput. 235, 168 - 173 (2014)

9. A. Ahmad, M. Arshad, G. Izarikova, AKCE Int. J. Graphs Comb. 12, $161-168$ (2015)

10. M. Bača, S. Jendrol, K. Kathiresan, K. Muthugurupackiam, A.S. Fenovcikova, Electron. Notes Discret. Math. 48, 19 - 26 (2015)

11. D. Indriati, Widodo, I.E. Wijayanti, K.A. Sugeng, M. Bača, Math. Comput. Sci. 9(2), $161-167$ (2015)

12. P. Jeyanthi, A. Sudha, Electron. Notes Discret. Math. 48, 175 - 182 (2015)

13. M.K. Siddiqui, D. Afzal, M.R. Faisal, J. Comb. Optim. 34, 534 - 544 (2017)

14. R.W. Putra, Y. Susanti, The total edge irregularity strength of uniform theta graphs, J. Phys. Conf. Ser. 1097, 0012069 (2018)

15. R.W. Putra, Y. Susanti, AKCE Int. J. Graphs Comb. 15, 7 - 13 (2018)

16. L. Ratnasari, S. Wahyuni, Y. Susanti, D.J.E. Palupi, B. Surodjo, Total edge irregularity strength of arithmetic book graphs, J. Phys. Conf. Ser. 1306, 012032 (2019)

17. L. Ratnasari, S. Wahyuni, Y. Susanti, D.J.E. Palupi, Total edge irregularity strength of book graphs and double book graphs, AIP Conf. Proc. 2192, 040013 (2019)

18. L. Ratnasari, Y. Susanti, Asian-European J. Math. 13, 2050072 (2020)

19. Y. Susanti, Y.I. Puspitasari, H. Khotimah, Iran. J. Math. Sci. Inform. 15, 1- 13 (2020)

20. L. Ratnasari, S. Wahyuni, Y. Susanti, D.J.E. Palupi, Total edge irregularity strength of triple book graphs, J. Phys. Conf. Ser. 1772(1), (2020) (Accepted) 